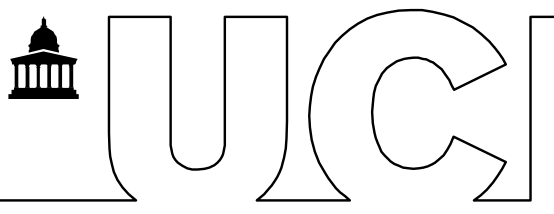

Payongsri, P; Steadman, D; Hailes, HC; Dalby, PA; (2015) Second generation engineering of transketolase for polar aromatic aldehyde substrates. Enzyme and Microbial Technology, 71 pp. 4552. 10.1016/..enzmictec.2015.01.008. Downloaded from UCL Discovery: http://discovery.ucl.ac.uk/1464511

\title{
ARTICLE
}

\section{Supplementary Data: Second Generation Engineering of Transketolase for Polar Aromatic Aldehyde Substrates}

\author{
Panwajee Payongsri, David Steadman ${ }^{b}$, Helen C. Hailes ${ }^{b}$, Paul A. Dalby ${ }^{a,{ }^{*}}$ \\ ${ }^{a}$ Department of Biochemical Engineering, University College London, Gordon Street, \\ London, WC1H OAH, UK. \\ ${ }^{b}$ Department of Chemistry, University College London, 20 Gordon Street, London WC1H \\ OAJ, UK. \\ "Corresponding author: Professor Paul A. Dalby, Department of Biochemical Engineering, \\ University College London, Gordon Street, London, WC1H OAH, UK; Email: \\ p.dalby@ucl.ac.uk
}

\section{Additional experimental details}

HPLC analysis of 3-FBA and 4-FBA reaction samples

Samples were analysed by HPLC as previously described [33] on an ACE5 C18 reverse phase column (150x4.6 mm), two mobile phases $0.1 \%$ TFA and $100 \%$ acetonitrile, and a flow rate of $1 \mathrm{ml} / \mathrm{min}$. The flow was started at $85 \%$ TFA $(0.1 \%)$ and reduced to $28 \%$ over 9 mins, followed by 2 mins equilibration at $85 \%$ TFA $(0.1 \%)$ and $15 \%$ acetonitrile. The retention times of 3-(1,3-Dihydroxy-2-oxopropyl) benzoic acid, 3-FBA, 4-(1,3-Dihydroxy-2oxopropyl) benzoic acid and 4-FBA were 2.97, 5.47, 2.45 and 5.41 mins, respectively.

Computational docking of 3-FBA, 4-FBA and 3-HBA in transketolase variant active-sites The structures of TK variants, aldehydes and ThDP enamine were modelled as previously described (Payongsri et al, 2012). TK variant structures were prepared by replacing the target residues of the E. coli wild type (PDB ID: 1QGD) (Littlechild et al, 1995). Residues within $10 \AA$ from the ThDP molecule were selected and subjected to energy minimised by Discovery Studio 2.0 (Accelrys, Inc. San Diego, California, USA). The energy minimised was performed for 1000 steps by CharmM Forcefield, with Adopted Basic NR, an implicit generalised Born solvent model, and True SHAKE constant (Payongsri et al, 2012). ThDPenamine and all the aldehyde substrates were drawn in chem3D ultra v.10. They were then energy minimised by MM2 calculation. The energy minimised ThDP-enamine structure was then docked into the active site of the energy-minimised TK variants prepared as above using. The aldehydes were then docked into these ThDP-enamine-TK structures. All computational docks were performed by AutoDock 4.2 and AutoDock Tools 1.5.4 (Goodsell et al, 1996). The centre of all the variants for docking the aldehydes was 11.777, 27.078, 37.195 with the grid size of $30 \AA \times 30 \AA \times 30 \AA$.

Validation of using whole cells in library screening The conversion to product obtained with both whole cells and clarified lysates, after 18 hours reaction with 4-FBA and 3-HBA, are compared in Fig. S1 and were found to correlate linearly. This demonstrated that the screening assay using whole cells adequately represents the activities of the enzymes obtained in clarified lysates as they would be used 
in an industrial biocatalytic process. Conversions after 18 hours using whole cells were approximately $50 \%$ of those using clarified lysates, suggesting that mass-transfer of the aromatic substrates into the whole cells partially slows the rate and hence conversions observed.

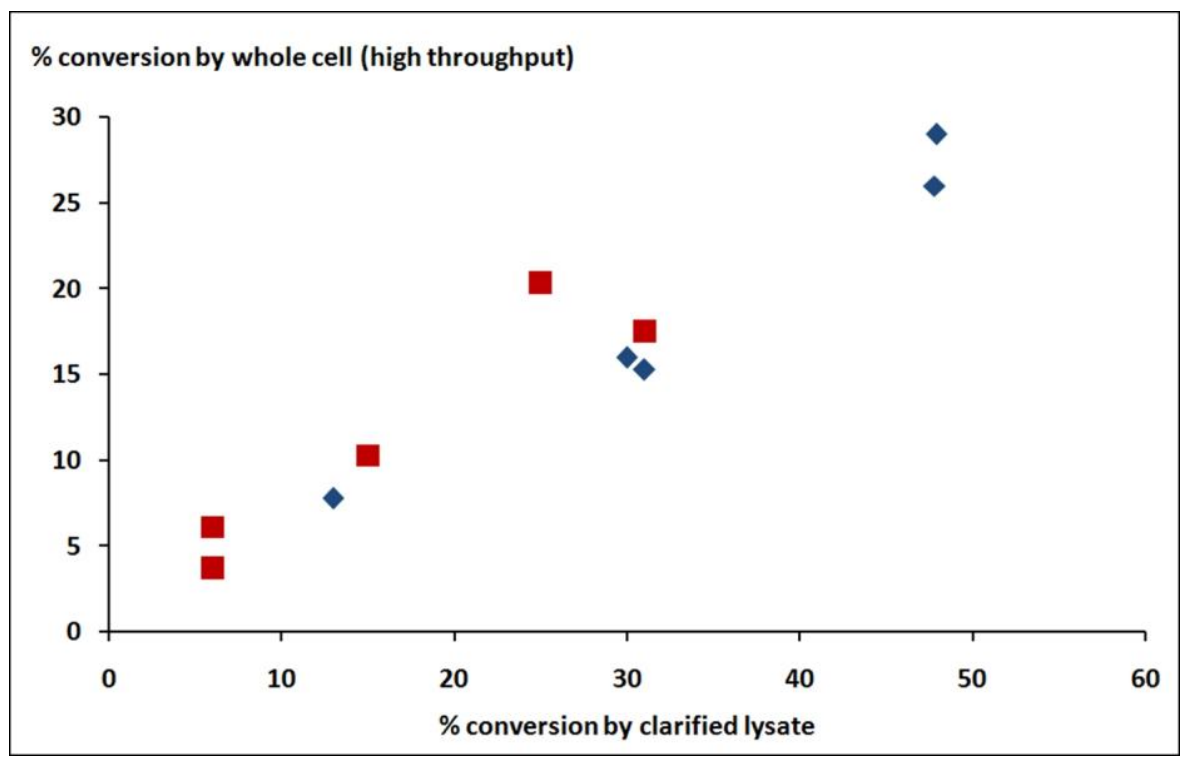

Fig. S1. Comparison of product conversions obtained with 4-FBA and 3-HBA in whole cells and with clarified lysates.

Percentage conversion to product obtained with clarified lysate and whole cells after 18 hours of reaction are compared for both 4-FBA $>$ and 3-HBA $\square$.

For 3-FBA, reactions were considerably faster than for 4-FBA or 3-HBA, and so activities towards 3-FBA could be determined directly from conversions at an earlier time-point (1 hour), using whole cells. For example, after 1 hour the conversion by D469T is $<35 \%$. Seven variants selected randomly from the new library were analysed in detail with 3-FBA and showed that the specific activity in clarified lysates correlated well with those determined using whole cells in the library screen (Fig. S2). At the highest specific activity reached, mass-transfer into whole cells appeared to have become limiting for 3-FBA. 


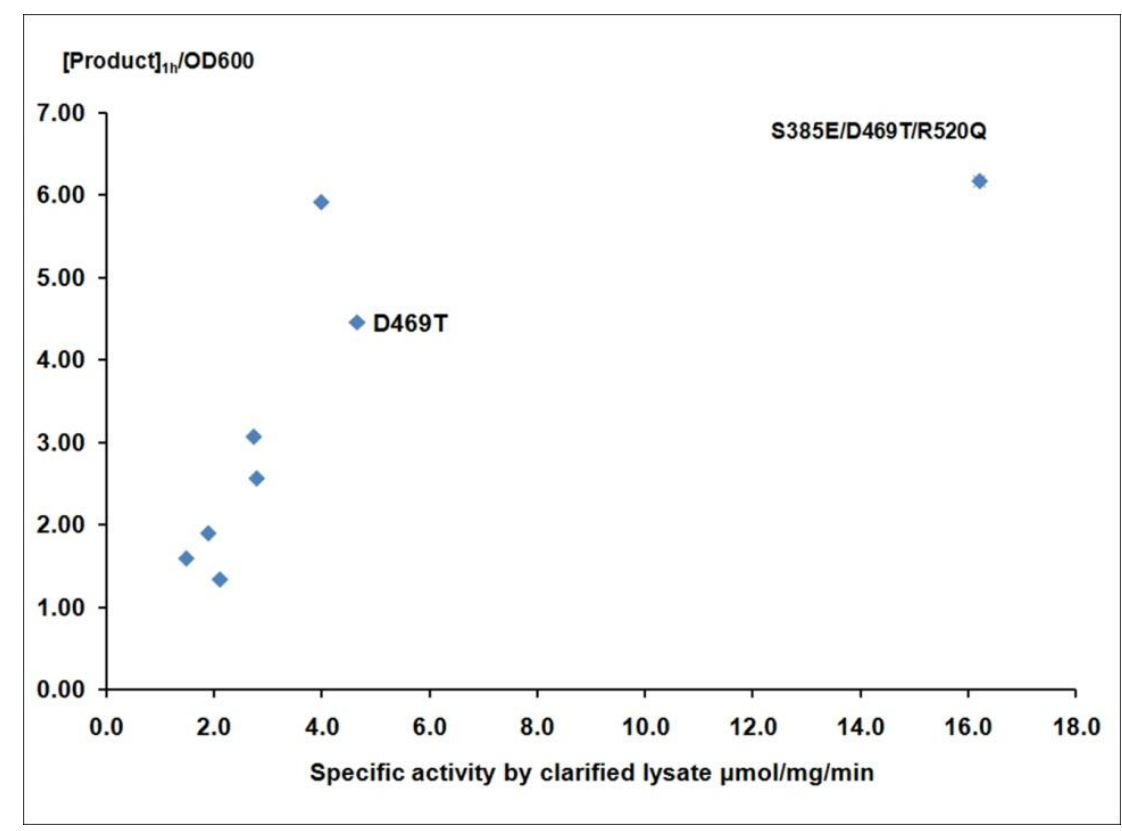

Fig. S2. Comparison of activities towards 3-FBA in the whole cell, with specific activities in clarified lysates.

The activities in whole cells were obtained by dividing the \% conversion to product obtained after 1 hour, by the OD600 of the cell culture used in each case.

\section{Correlation of specific activity and yield in clarified lysates}

After screening all three aromatic aldehyde substrates on both the S385X/D469T/R520Q and R358X/D469T/R520Q libraries with whole cells, variants were selected randomly across the range from low to high activity for a more detailed analysis of specific activities and yields obtained with clarified lysates. The correlation between specific activity and conversion to product after 18 hours remained true for 4-FBA and 3-HBA with five variants selected randomly from the new library (Fig. S3).

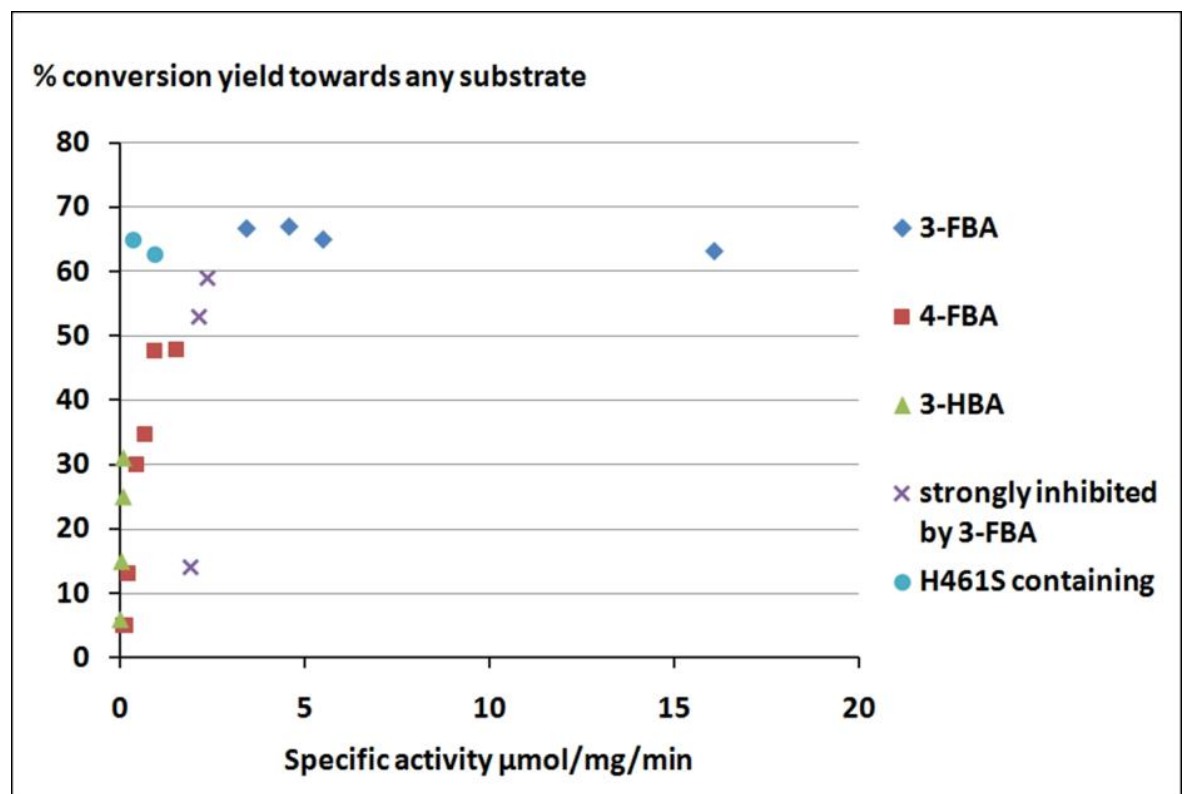

Fig. S3. The relationship between specific activities and the \% conversion to product at 18 hours for different aromatic aldehydes.

Previously identified D469T-containing variants are shown alongside five variants newly selected in the present study for increased activity towards 4-FBA and 3-HBA. 
The reactions with 3-FBA are generally faster than those with 4-FBA and 3-HBA and a maximum yield is reached well before 18 hours for $3-F B A$. The conversions to product obtained after 18 hours for 4-FBA and 3-HBA tend to increase with specific activity, as the product formation out-competes any HPA substrate degradation. All variants were found previously to be inhibited by 3-FBA substrate to some degree. This accounts for the maximum conversion of less than $100 \%$, as well as the outlier below the main curve with a much lower than expected conversion yield. The two variants H461S/D469T and $\mathrm{H} 461 \mathrm{~S} / \mathrm{D} 469 \mathrm{~T} / \mathrm{R} 520 \mathrm{Q}$ also did not fit the overall trend. These two variants were found to be unstable, with impaired apparent $k_{\text {cat }}$-values.

\section{Enzyme stability during 18-hour reactions}

It is possible that a correlation between specific activity and conversion at 18 hours could result from enzyme stability loss over the 18 hours. This was assessed for S385E/D469T/R520Q under 2 conditions. In condition 1, six aliquots of lysate with cofactor were incubated with 3-FBA in the absence of HPA. Reactions were then started by addition of HPA at $0,2,3,4,5$, and 21 hours. In condition 2, six aliquots of lysate with cofactor were left at $22{ }^{\circ} \mathrm{C}$ without addition of any substrate. Reactions were then started by addition of HPA and 3-FBA at 0, 2, 3, 4, 5, and 21 hours.

As shown in Fig. S4, the activity retained was more than $70 \%$ after 18 hours when no substrate was added. If 3-FBA was added, sharp drop in activity was observed in the first 2 hours to about $55 \%$, followed by a slow inactivation to $45 \%$ after 18 hours.

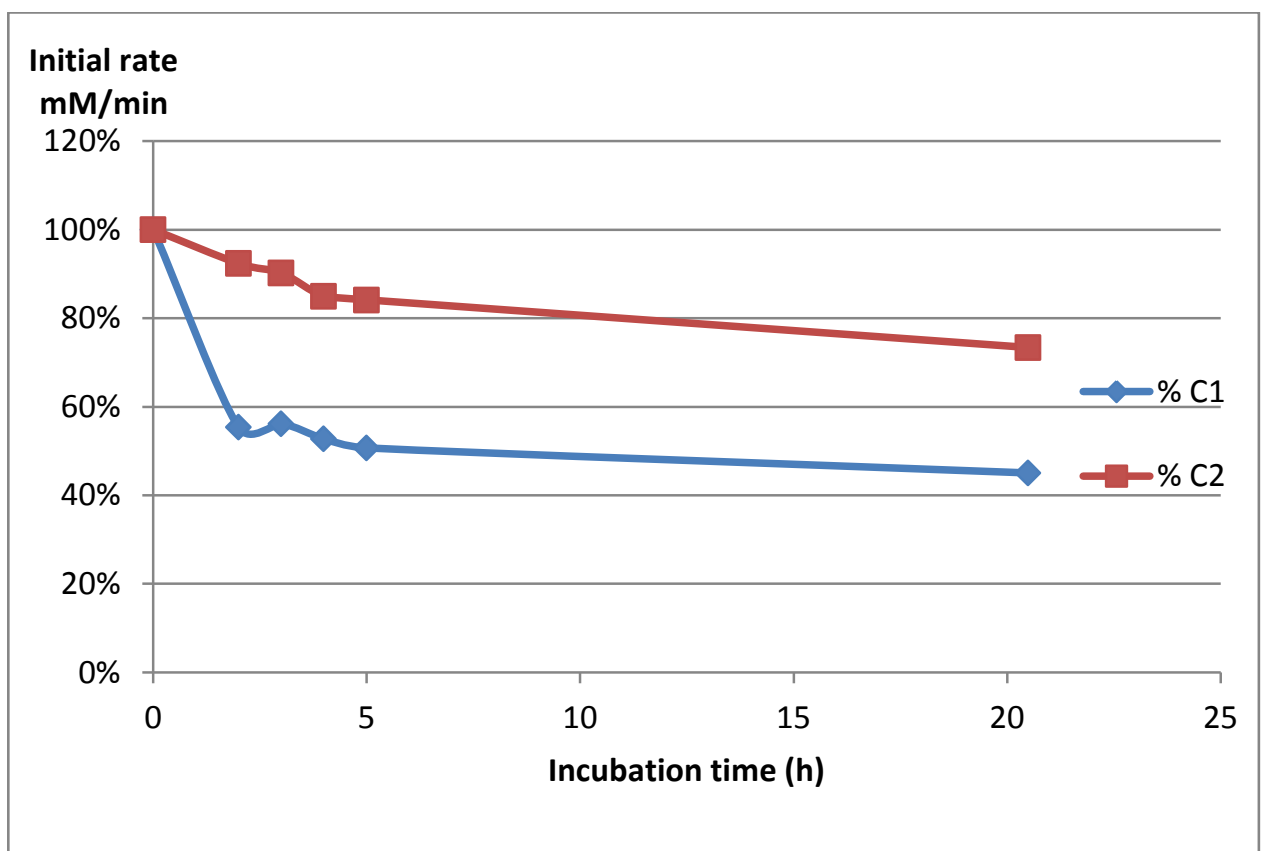

Fig. S4. Retention of TK enzyme variant S385E/D469T/R520Q activity over 21 hours of pre-incubation under reaction conditions, prior to activity assessment.

Condition $1(\mathrm{C} 1)$ : pre-incubation at $22^{\circ} \mathrm{C}$ in the absence of HPA. Condition 2 (C2): preincubation at $22^{\circ} \mathrm{C}$ in the absence of both 3-FBA and HPA.

\section{References}

D.S. Goodsell, G.M. Morris and A.J. Olson, Automated docking of flexible ligands: applications of AutoDock, J Mol Recognit 9, 1996, 1-5

Payongsri P, Steadman D, Strafford J, MacMurray A, Hailes HC, Dalby PA. Rational substrate and enzyme engineering of transketolase for aromatics. Org Biomol Chem 2012;10:9021-9. 
Littlechild J, Turner N, Hobbs G, Lilly M, Rawas A, Watson H. Crystallization and preliminary X-ray crystallographic data with Escherichia coli transketolase. Acta Crystallog Sect D: Biol Crystallog 1995;51:1074-6. 